\title{
UTILIZAÇÃO DE FERRAMENTAS BIM NA AVALIAÇÃO DA EFICIÊNCIA ENERGÉTICA DOS EDIFÍCIOS - ESTADO DA ARTE
}

\author{
José Santos ${ }^{(1,2, *)}$, Patrícia Escórcio ${ }^{(1)}$, Vítor Pereira ${ }^{(3)}$ \\ (1) Universidade da Madeira, Faculdade de Ciências Exatas e da Engenharia, Departamento de \\ Engenharia Civil e Geologia, 9020-105 Funchal \\ (2) CONSTRUCT-LABEST, Faculdade de Engenharia (FEUP), Universidade do Porto \\ (3) Universidade da Madeira (Mestrando) \\ (*)jmmns@fe.up.pt
}

\begin{abstract}
Resumo
$\mathrm{Na}$ última década as exigências relativas à eficiência energética dos edifícios aumentaram substancialmente. Por sua vez, as ferramentas BIM têm permitido a realização de tarefas de otimização de soluções na fase de conceção que antes eram muito trabalhosas, assim como difíceis de conciliar. Neste artigo faz-se uma revisão bibliográfica sobre a utilização de ferramentas BIM com a finalidade de obter edifícios energeticamente mais eficientes. São identificados e analisados os tópicos mais relevantes sobre o tema, assim como identificadas as ferramentas BIM mais utilizadas. Constata-se que embora haja ainda muitos aspetos a melhorar, já é possível usar as ferramentas BIM para obter edifícios mais eficientes.
\end{abstract}

\section{Introdução}

O setor da construção representa $40 \%$ do consumo total de energia na UE. Por isso, os diversos países estão a tomar medidas para que os novos edifícios tenham necessidades quase nulas de energia (NZEB) e os edifícios existentes se aproximem dos NZEB à medida que são reabilitados. A avaliação da eficiência energética dos edifícios é realizada através da comparação das propriedades térmicas dos elementos construtivos, da eficiência dos sistemas eletromecânicos e das necessidades energéticas dos edifícios, com valores de referência.

Neste contexto, dado que o modelo BIM tem, em princípio, todas as informações necessárias para as avaliações a realizar, coloca-se a questão de saber até que ponto as ferramentas BIM estão a ser usadas para este fim, de que forma e a sua eficiência. Assim, este artigo tem como objetivo, através da realização de uma revisão bibliográfica sobre a aplicação de ferramentas BIM na avaliação da eficiência energética dos edifícios, responder a esta questão.

Para tal, na Secção 2 é exposta a metodologia seguida na realização deste trabalho, na Secção 3 é apresentado um resumo dos tópicos principais identificados na literatura e das ferramentas BIM existentes no contexto da eficiência energética, na Secção 4 são analisados em detalhe os tópicos identificados e, finalmente, na Secção 5 são apresentadas as conclusões obtidas. 


\section{Metodologia}

Na elaboração deste trabalho foi seguida a metodologia indicada na Figura 1.

A pesquisa bibliográfica decorreu nas bases de dados: Google Scholar, Science Direct, ASCE Library, ICE Virtual Library e Google com os termos "Building Information Modelling", "Energy Efficiency", "Energy performance", "Green Building" ou suas combinações, tendo resultado em cerca de 1350 documentos.

Seguidamente procedeu-se uma seleção dos artigos principais através da análise do resumo e conclusão dos mesmos, tendo sido excluídos os que de facto não se relacionavam com a utilização de BIM para realizar análises energéticas dos edifícios, seja sobre a forma de caso prático, artigo de desenvolvimento ou artigo de revisão, tendo resultado em cerca de 70 artigos. Note-se que houve muitos artigos de áreas com alguma sobreposição à analisada aqui, que abordam temas de elevado interesse como: a incorporação energética dos materiais de construção e gastos no seu fabrico (e não só os consumos do edifício ao longo da vida), os diversos impactes do edifício ao longo da vida (Avaliação do Ciclo de Vida) (e não só os consumos energéticos), análises que podem ser desenvolvidas com apoio de ferramentas BIM, mas que ultrapassam o âmbito deste artigo, e que por isso não foram considerados.

Posteriormente (ver Secção 3), os artigos selecionados foram analisados por completo, tendo em vista a identificação do tópico principal do artigo e das ferramentas BIM utilizadas. Após ponderação, selecionaram-se 16 tópicos e identificaram-se 18 ferramentas BIM.

Por último (ver Secção 4), para cada tópico identificado foi feito um breve resumo do trabalho desenvolvido e das conclusões obtidas pelos diversos autores.



Figura 1: Metodologia.

\section{Tópicos identificados na literatura e Ferramentas BIM}

Durante a leitura dos artigos selecionados foram identificados os seus tópicos principais, assim como foi feita uma listagem das ferramentas BIM (software) existentes.

\subsection{Tópicos identificados na literatura}

Na Tabela 1 apresentam-se os tópicos principais selecionadas e os respetivos artigos. Estes são agregados em três grandes grupos:

i) os que se focam na tecnologia BIM (Junção de nuvens de pontos do laser scanner com pontos da termografia, Interoperabilidade, Parâmetros IFC e Bibliotecas BIM);

ii) os que se focam na eficiência energética (Conforto térmico, Certificação Energética, NZEB, Reabilitação de edifícios, Edifícios industriais, Análises ao nível da cidade); 
iii) os que se focam no uso BIM para eficiência energética (Identificação das melhores soluções técnicas ao nível de materiais, janelas, fachadas, orientações, etc. tendo em vista a redução de consumos energéticos dos edifícios, Estimativa dos consumos energéticos dos edifícios, Utilização do BIM como plataforma para a visualização de resultados, Recolha de dados técnicos (geometria, materiais, etc.) para o cálculo de parâmetros e índices energéticos noutras plataformas, Desenvolvimento de software e plugins, Sensores e Monitorização, Ensino).

Tabela 1: Tópicos identificados na literatura

\begin{tabular}{lc}
\hline Tópicos & Artigos \\
\hline Junção de nuvens de pontos do laser scanner e da termografia & {$[1-9]$} \\
Interoperabilidade & {$[10-15]$} \\
Parâmetros IFC / Bibliotecas BIM & {$[11,14,16,17]$} \\
\hline Conforto térmico & {$[18-23]$} \\
Reabilitação de edifícios & {$[6,24-27]$} \\
Certificação Energética & {$[28-31]$} \\
Análises ao nível da cidade & {$[6,32,33]$} \\
NZEB & {$[14,34,35]$} \\
Edifícios industriais & {$[5,36]$} \\
\hline Comparação de soluções e otimização (materiais, janelas, fachadas, & {$[2,23,35,37-48]$} \\
orientações, etc.) & {$[34,37,43,48-52]$} \\
Estimativa de consumos & {$[33,49,53-57]$} \\
Plataforma de visualização de resultados & {$[34,40,43,50,51$,} \\
Recolha de informação automática para / cálculos / índices / & $58-60]$ \\
programação / simulações & {$[24,61-63]$} \\
Ensino / Qualidade do ensino & {$[64,65]$} \\
Desenvolvimento de software / plugins & {$[66]$} \\
Sensores / Monitorização & \\
\hline
\end{tabular}

\subsection{Ferramentas BIM}

Nas referências selecionadas foram utilizadas as seguintes ferramentas BIM: ArcGIS, Autodesk Ecotect Analysis (produto descontinuado), Autodesk Green Building Studio, Autodesk Insight, Autodesk Revit, Daysim, DesignBuilder, Energy Plus, eQUEST, Grasshopper 3D, IDA ICE, IES Virtual Environment, Rhinoceros 3D, Sefaira, SketchUp, Tally Environmental Impact Tool, TRNSYS, 6SigmaDC.

Constatou-se que na maioria das situações foi utilizada a ferramenta Autodesk Green Building Studio, o que se pode justificar por diversos motivos técnicos e/ou comerciais. Devido à limitação do número de páginas deste artigo não é possível desenvolver esta secção, no entanto refira-se que existem muitas outras ferramentas disponíveis, pelo que se recomenda a consulta de $[67,68]$ onde existe uma vasta lista de ferramentas disponíveis e organizadas por categorias. 


\section{Estado de Arte de cada tópico identificado na literatura}

Com base nos artigos indicados na Tabela 1 apresentam-se a seguir as ideias chave sobre cada um dos tópicos identificados na literatura. Devido à limitação do número de páginas apenas se abordam os 10 tópicos com mais artigos.

\subsection{Junção de dados laser scanner com dados da termografia}

Neste tópico tem-se por objetivo juntar os dados das nuvens de pontos 3D com a termografia de modo a que cada ponto 3D do edifício contenha também, pelo menos, a informação da temperatura. Isto tem utilidade em edifícios existentes aparecendo, portanto, associada à reabilitação de edifícios.

Diversas soluções de software e hardware foram apresentadas para processar automaticamente os dois tipos de dados num único ficheiro (geralmente no formato gbXML). Refira-se que atualmente este trabalho deixou de ser necessário, uma vez que já existem laser scanners que incorporam a termografia, como o BLK360 da Leica-Geosystems.

Existem duas utilidades deste tópico: i) a calibração dos modelos BIM, dado que em muitos edifícios existentes se desconhece com rigor as propriedades térmicas dos materiais e influência das pontes térmicas, e ii) estimativa do coeficiente de transmissão térmica dos elementos. Sobre estes dois assuntos existe ainda pouco trabalho desenvolvido, em comparação com o apresentado no parágrafo anterior, esperando-se novas aplicações no futuro, uma vez que já existe conhecimento suficiente ao nível da calibração de modelos e diversos métodos indiretos de estimativa do coeficiente de transmissão térmica.

\subsection{Interoperabilidade}

Neste tópico tem-se por objetivo que exista interoperabilidade não só entre ferramentas BIM na fase de projeto, mas também durante todo o ciclo de vida do edifício, de modo a que não haja perdas de informação e que toda a informação possa ser adequadamente usada em todas as ferramentas.

Foram encontrados dois tipos de trabalhos: i) os que apenas demonstram com casos práticos, através do uso de ferramentas existentes, todos os passos necessários, desde a modelação até aos consumos energéticos, para executar uma análise energética, e ii) os que desenvolvem API e/ou plugins que leem, tratam (adicionam, corrigem, etc.) a informação de uma dada ferramenta e a disponibilizam num formato capaz de ser lido por outra aplicação.

Espera-se que o formato IFC4 com a MVD Energy Analysis View possa melhorar a interoperabilidade entre ferramentas.

\subsection{Conforto térmico}

O conforto térmico resulta da análise de seis parâmetros, dos quais dois dependem do indivíduo (atividade metabólica e resistência térmica da roupa) e os restantes quatro dependem da conceção do edifício (temperatura do ar, temperatura de radiação, humidade e velocidade do ar).

Neste tópico o objetivo passa por usar o modelo BIM para estimar os últimos quatro parâmetros acima referidos. Para tal, além das ferramentas BIM de modelação, que permitem estimar a radiação solar, o modelo BIM pode ser exportado para ferramentas de análise energética, para estimar a temperatura e humidade, assim como para ferramentas de dinâmica de fluidos (CFD) 
para estimar a velocidade do vento (quando se usa ventilação natural). Deste modo é possível estimar os dois índices de conforto térmico ao longo de um ano, nomeadamente PMV e o PPD.

\subsection{Reabilitação de edifícios}

Neste tópico existem diversos objetivos: i) levantamento do edifício, recorrendo ao laser scanner, tratamento de nuvens de pontos para definição da geometria; ii) estudo das soluções ideais de reabilitação (portanto sem alteração da geometria) para casos concretos ou para casos genéricos (medidas tipo); iii) comparação de consumos (antes e depois da reabilitação) com o estimado nos modelos iniciais, iv) avaliação de soluções passivas versus soluções ativas, principalmente em edifícios históricos.

\subsection{Certificação Energética}

Neste tópico, além do que será referido na Secção 4.9 relativamente à possibilidade de obtenção automática de dados para usar no cálculo de índices energéticos e ambientais, refere-se que: i) no futuro as certificações energéticas e ambientais serão realizadas automaticamente através do modelo BIM e, ii) a existência de milhares de modelos BIM permitirá através de técnicas de big data melhorar a construção, a reabilitação e a própria certificação.

\subsection{Comparação de soluções e otimização}

Neste tópico a utilização do BIM tem como objetivo atingir a solução que despenda menos energia ao longo do ciclo de vida do edifício. Para tal existem três fases conceptuais: a elaboração do modelo BIM, exportação para um modelo BEM (Building Energy Model) e cálculo energético. Estas fases podem ser feitas num único software com recurso a plugins ou realizado em softwares diferentes.

As análises mais simples baseiam-se na: i) criação de diferentes cenários (soluções alternativas de materiais, janelas, fachadas, orientações, áreas, etc.), ii) cálculo dos consumos energéticos e iii) escolha da melhor solução. As análises mais avançadas envolvem alguma programação visual (ex: Dynamo, Grasshopper) ou programação por código, onde alguns parâmetros do edifício podem ser variáveis e onde são implementados algoritmos de otimização para atingir a melhor solução (normalmente o menor consumo energético), sendo os algoritmos mais utilizados atualmente baseados em Algoritmos Genéticos e/ou no Método de Monte Carlo.

\subsection{Estimativa de consumos}

Neste tópico o objetivo principal consistiu na explicação do processo de criação do modelo BIM e realização das análises energéticas, com o objetivo de demonstrar a facilidade com que atualmente os arquitetos, maioritariamente, podem usar estas ferramentas para numa fase preliminar obter edifícios mais eficientes. A ferramenta de simulação mais referida na literatura é o Autodesk Green Building Studio. Um dos inconvenientes é que estas ferramentas não incluem ainda normas portuguesas pelo que os seus resultados só podem ser analisados em termos comparativos ou qualitativos.

\subsection{Plataforma de visualização de resultados}

Neste tópico as ferramentas BIM são usadas em conjunto com outras ferramentas diferentes, como por exemplo: de planeamento urbano (SIG), de manutenção de edifícios, de projeto (térmico, ventilação, iluminação), etc. sendo que os resultados das previsões ou os resultados reais de consumos são mostrados em modelos BIM, para fácil perceção dos utilizadores. 
$\mathrm{Na}$ maioria das situações esta visualização ocorre através da atribuição de diferentes cores a espaços e/ou objetos, sendo a informação transmitida ao modelo BIM através de parâmetros partilhados.

Sendo a visualização por cores apenas uma das formas de ver resultados e a troca de informações referida pouco robusta para grandes quantidades de informação, percebe-se que esta é uma área ainda em desenvolvimento, esperando-se novidades nos próximos anos, embora se reconheça que esta não é a função principal das ferramentas BIM clássicas.

\subsection{Recolha de informação automática para cálculos e parâmetros}

Neste tópico o objetivo principal passa pela redução do tempo necessário para a execução de tarefas que realizadas manualmente requereriam muitas horas de trabalho. Algumas das vantagens da maioria das ferramentas BIM é que é possível: transferir informação dinamicamente, interagir entre ferramentas, criar novas ferramentas, APIs ou plugins, programar dentro das próprias ferramentas com linguagens visuais, etc.

Ora, isto abre um campo de possibilidades extremamente vasto contribuindo para a proliferação de pequenos softwares e/ou plugins capazes de realizar tarefas muito especificas. Com o passar do tempo, os mais interessantes acabam por ser incorporados nas ferramentas BIM tradicionais. Desde modo, para o tema da eficiência energética dos edifícios observa-se: o desenvolvimento de múltiplos modelos de otimização (para minimização de consumos, obtenção de máxima classificação em índices green, etc.), a extração automática de informação para ferramentas próprias ou oficiais e a possibilidade de certificação energética.

\subsection{Ensino}

Neste tópico, além do que já foi referido na Secção 4.7 relativamente a diversos exemplos a explicar todo o processo de cálculo, constata-se uma muito baixa implementação do uso de ferramentas BIM para realização de cálculos energéticos dinâmicos pois não só as ferramentas são ainda relativamente recentes, como não era hábito dos arquitetos e engenheiros civis realizar tais cálculos, uma vez que os engenheiros mecânicos é que projetam o AVAC, mas que por sua vez ainda estão pouco vocacionados para o uso do BIM. Deste modo, é de salientar o projeto europeu BIMcert, que pretendeu ajudar a mitigar algumas destas deficiências.

\section{Conclusões}

A avaliação da eficiência energética dos edifícios utilizando ferramentas BIM tem evoluído bastante nos últimos anos, esperando-se que a curto prazo a forma como arquitetos e engenheiros projetam os edifícios logo no início da sua conceção (onde as decisões fundamentais são tomadas) seja ambientalmente mais responsável.

Ao nível da tecnologia BIM têm-se registado evoluções importantes ao nível da recolha de nuvem de pontos e software, mas verificam-se ainda dificuldades ao nível da interoperabilidade. Ao nível da eficiência energética, existem novas potencialidades, como a avaliação do conforto térmico, esperando-se que no futuro a certificação energética seja realizada automaticamente. Ao nível do uso do BIM para avaliar e melhorar o desempenho energético dos edifícios, já é possível usar algumas ferramentas para, através da realização de múltiplas simulações, obter soluções otimizadas, ainda que os resultados possam não ser rigorosamente aplicáveis à realidade normativa portuguesa (clima, materiais, etc.). 


\section{Agradecimentos}

Este trabalho foi financiado por: Financiamento Base - UIDB/04708/2020 da Unidade de Investigação CONSTRUCT - Instituto de I\&D em Estruturas e Construções - financiada por fundos nacionais através da FCT/MCTES (PIDDAC).

\section{Referências}

[1] Lagüela, S., et al., Automatic thermographic and RGB texture of as-built BIM for energy rehabilitation purposes. Automation in Construction, 2013. 31: p. 230-240.

[2] Roslan, R., et al., Building Information Modelling (BIM) For Estimation of Heat Flux from Streetscape Material. International Journal of Engineering and Advanced Technology, 2019. 9(1): p. 3520-3524.

[3] Garwood, T.L., et al., A framework for producing gbXML building geometry from Point Clouds for accurate and efficient Building Energy Modelling. Applied Energy, 2018. 224: p. 527-537.

[4] Cho, Y. and C. Wang, 3D Thermal Modeling for Existing Buildings Using Hybrid LIDAR System, in Computing in Civil Engineering (2011). 2011. p. 552-559.

[5] Garwood, T.L., et al., Geometry Extraction for High Resolution Building Energy Modelling Applications from Point Cloud Data: A Case Study of a Factory Facility. Energy Procedia, 2017. 142: p. 1805-1810.

[6] Gigliarelli, E., F. Calcerano, and L. Cessari, Implementation Analysis and Design for Energy Efficient Intervention on Heritage Buildings, in Digital Heritage. Progress in Cultural Heritage: Documentation, Preservation, and Protection. 2016. p. 91-103.

[7] Ham, Y. and M. Golparvar-Fard, Mapping actual thermal properties to building elements in gbXML-based BIM for reliable building energy performance modeling. Automation in Construction, 2015. 49: p. 214-224.

[8] Ham, Y. and M. Golparvar-Fard, An automated vision-based method for rapid 3D energy performance modeling of existing buildings using thermal and digital imagery. Advanced Engineering Informatics, 2013. 27(3): p. 395-409.

[9] Wang, C. and Y.K. Cho, Performance Evaluation of Automatically Generated BIM from Laser Scanner Data for Sustainability Analyses. Procedia Engineering, 2015. 118: p. 918925.

[10] Muller, M.F., et al., A systematic literature review of interoperability in the green Building Information Modeling lifecycle. Journal of Cleaner Production, 2019. 223: p. 397-412.

[11] Kim, J.B., et al., Developing a physical BIM library for building thermal energy simulation. Automation in Construction, 2015. 50: p. 16-28.

[12] Arayici, Y., et al., Interoperability specification development for integrated BIM use in performance based design. Automation in Construction, 2018. 85: p. 167-181.

[13] O'Donnell, J., et al., Transforming BIM to BEM: generation of building geometry for the NASA Ames sustainability base BIM. 2013, LBNL-6033E, University of California, Berkeley.

[14] Spiridigliozzi, G., et al., BIM-BEM support tools for early stages of zero-energy building design. IOP Conference Series: Materials Science and Engineering, 2019. 609: p. 072075. 
[15] Ying, H. and S. Lee, An algorithm to facet curved walls in IFC BIM for building energy analysis. Automation in Construction, 2019. 103: p. 80-103.

[16] Maltese, S., et al., Sustainability Assessment through Green BIM for Environmental, Social and Economic Efficiency. Procedia Engineering, 2017. 180: p. 520-530.

[17] Mahmoud, S., T. Zayed, and M. Fahmy, Development of sustainability assessment tool for existing buildings. Sustainable Cities and Society, 2019. 44: p. 99-119.

[18] Habibi, S., The promise of BIM for improving building performance. Energy and Buildings, 2017. 153: p. 525-548.

[19] Gan, V.J.L., et al., BIM-based framework to analyze the effect of natural ventilation on thermal comfort and energy performance in buildings. Energy Procedia, 2019. 158: p. 3319-3324.

[20] Oduyemi, O. and M. Okoroh, Building performance modelling for sustainable building design. International Journal of Sustainable Built Environment, 2016. 5(2): p. 461-469.

[21] Sultanguzin, I.A., et al., Using of BIM, BEM and CFD technologies for design and construction of energy-efficient houses. E3S Web Conf., 2019. 124: p. 03014.

[22] Coakley, D., E. Corry, and M. Keane. Validation of Simulated thermal comfort using a calibrated building energy simulation (BES) model in the context of building performance evaluation \& optimisation. in 13th Annual International Conference for Enhanced Building Operations (ICEBO). 2013. Energy Systems Laboratory, Texas A\&M University.

[23] He, B.-j., et al., The combination of digital technology and architectural design to develop a process for enhancing energy-saving: The case of Maanshan China. Technology in Society, 2014. 39: p. 77-87.

[24] Latif, A.F., et al., A Review on Energy Performance in Malaysian Universities Through Building Information Modelling (BIM) Adaptation. IOP Conference Series: Earth and Environmental Science, 2019. 291: p. 012033.

[25] Capeluto, I.G. and C.E. Ochoa, Simulation-based method to determine climatic energy strategies of an adaptable building retrofit façade system. Energy, 2014. 76: p. 375-384.

[26] Spiegelhalter, T., Energy-efficiency Retrofitting and Transformation of the FIU-college of Architecture + The Arts into a Net-Zero-Energy-Building by 2018. Energy Procedia, 2014. 57: p. 1922-1930.

[27] Mytafides, C.K., A. Dimoudi, and S. Zoras, Transformation of a university building into a zero energy building in Mediterranean climate. Energy and Buildings, 2017. 155: p. 98114.

[28] Liu, Z., et al., A feasibility study of Building Information Modeling for Green Mark New Non-Residential Building (NRB): 2015 analysis. Energy Procedia, 2017. 143: p. 80-87.

[29] Chen, P.-H. and T.C. Nguyen, Integrating web map service and building information modeling for location and transportation analysis in green building certification process. Automation in Construction, 2017. 77: p. 52-66.

[30] Jalaei, F. and A. Jrade, Integrating building information modeling (BIM) and LEED system at the conceptual design stage of sustainable buildings. Sustainable Cities and Society, 2015. 18: p. 95-107.

[31] Li, Y., et al., Review of building energy performance certification schemes towards future improvement. Renewable and Sustainable Energy Reviews, 2019. 113.

[32] Hong, T., et al., Ten questions on urban building energy modeling. Building and Environment, 2020. 168. 
[33] Niu, S., W. Pan, and Y. Zhao, A BIM-GIS Integrated Web-based Visualization System for Low Energy Building Design. Procedia Engineering, 2015. 121: p. 2184-2192.

[34] Chen, S.Y., Use of Green Building Information Modeling in the Assessment of Net Zero Energy Building Design. Journal of Environmental Engineering and Landscape Management, 2019. 27(3): p. 174-186.

[35] Najjar, M., K. Figueiredo, and A. Haddad, Increasing energy efficiency of building envelopes towards nearly zero energy buildings integrating BIM and LCA, in LA SDEWES 2018 - 1st Latin American Conference on sustainable development of energy, water and environment systems. 2018: Rio de Janeiro, Brasil.

[36] Gourlis, G. and I. Kovacic, Building Information Modelling for analysis of energy efficient industrial buildings - A case study. Renewable and Sustainable Energy Reviews, 2017. 68: p. 953-963.

[37] Luziani, S. and B. Paramita, Autodesk Green Building Studio an Energy Simulation Analysis in the Design Process. KnE Social Sciences, 2019.

[38] Sampaio, A.Z., L. Araújo, and E. Coelho, Building Information Modelling Capability in an Energetic Simulation Perspective, in INCREaSE 2019 - International Congress on Engineering and Sustainability in the XXI Century. 2019: Faro, Portugal. p. 465-477.

[39] Abanda, F.H. and L. Byers, An investigation of the impact of building orientation on energy consumption in a domestic building using emerging BIM (Building Information Modelling). Energy, 2016. 97: p. 517-527.

[40] Chen, L. and W. Pan, A BIM-integrated Fuzzy Multi-criteria Decision Making Model for Selecting Low-Carbon Building Measures. Procedia Engineering, 2015. 118: p. 606-613.

[41] Acosta, I., M.Á. Campano, and J.F. Molina, Window design in architecture: Analysis of energy savings for lighting and visual comfort in residential spaces. Applied Energy, 2016. 168: p. 493-506.

[42] Marzouk, M., S. Azab, and M. Metawie, BIM-based approach for optimizing life cycle costs of sustainable buildings. Journal of Cleaner Production, 2018. 188: p. 217-226.

[43] Rahmani Asl, M., et al., BPOpt: A framework for BIM-based performance optimization. Energy and Buildings, 2015. 108: p. 401-412.

[44] Chang, S., et al., Framework for evaluating and optimizing algae façades using closedloop simulation analysis integrated with BIM. Energy Procedia, 2017. 143: p. 237-244.

[45] Hamedani, M.N. and R.E. Smith, Evaluation of Performance Modelling: Optimizing Simulation Tools to Stages of Architectural Design. Procedia Engineering, 2015. 118: p. 774-780.

[46] Hiyama, K., et al., A new method for reusing building information models of past projects to optimize the default configuration for performance simulations. Energy and Buildings, 2014. 73: p. 83-91.

[47]Deepa, K., et al., Energy Analysis Of Buildings. International Research Journal of Engineering and Technology, 2019. 6(1): p. 1662-1666.

[48] Shoubi, M.V., et al., Reducing the operational energy demand in buildings using building information modeling tools and sustainability approaches. Ain Shams Engineering Journal, 2015. 6(1): p. 41-55.

[49] Cecchini, C., A. Magrini, and L. Gobbi, A 3d platform for energy data visualization of building assets. IOP Conference Series: Earth and Environmental Science, 2019. 296: p. 012035 . 
[50] Serra, E.G. and Z.R.P. Filho, Methods for Assessing Energy Efficiency of Buildings. Journal of Sustainable Development of Energy, Water and Environment Systems, 2019.

[51] Gao, H., et al., BIM-based real time building energy simulation and optimization in early design stage. IOP Conference Series: Materials Science and Engineering, 2019. 556: p. 012064.

[52] Singh, P. and A. Sadhu, Multicomponent energy assessment of buildings using building information modeling. Sustainable Cities and Society, 2019. 49.

[53] Abaglo, A.J., C. Bonalda, and E. Pertusa, Environmental Digital Model: Integration of BIM into environmental building simulations. Energy Procedia, 2017. 122: p. 1063-1068.

[54] Gerrish, T., et al., BIM application to building energy performance visualisation and management: Challenges and potential. Energy and Buildings, 2017. 144: p. 218-228.

[55] Bonenberg, W. and X. Wei, Green BIM in Sustainable Infrastructure. Procedia Manufacturing, 2015. 3: p. 1654-1659.

[56] Truong, H., et al., Method for visualizing energy use in building information models. Energy Procedia, 2017. 142: p. 2541-2546.

[57] Motawa, I. and K. Carter, Sustainable BIM-based Evaluation of Buildings. Procedia Social and Behavioral Sciences, 2013. 74: p. 419-428.

[58] Shadram, F. and J. Mukkavaara, An integrated BIM-based framework for the optimization of the trade-off between embodied and operational energy. Energy and Buildings, 2018. 158: p. 1189-1205.

[59] Ilhan, B. and H. Yaman, Green building assessment tool (GBAT) for integrated BIM-based design decisions. Automation in Construction, 2016. 70: p. 26-37.

[60] Najjar, M., et al., Integrated optimization with building information modeling and life cycle assessment for generating energy efficient buildings. Applied Energy, 2019. 250: p. 13661382.

[61] McAuley, B., et al., Delivering Energy Savings for the Supply Chain Through Building Information Modelling as a Result of the Horizon 2020 Energy BIMcert Project, in SEEDS 2019 - 5th International Conference SEEDS (Sustainable Ecological Engineering Design for Society). 2019: Ipswich, UK.

[62] El-Diraby, T., T. Krijnen, and M. Papagelis, BIM-based collaborative design and sociotechnical analytics of green buildings. Automation in Construction, 2017. 82: p. 59-74.

[63] Doan, D.T., et al., Examining Green Star certification uptake and its relationship with Building Information Modelling (BIM) adoption in New Zealand. Journal of Environmental Management, 2019. 250.

[64] Kamel, E. and A.M. Memari, Automated Building Energy Modeling and Assessment Tool (ABEMAT). Energy, 2018. 147: p. 15-24.

[65] Abdelalim, A., W. O'Brien, and Z. Shi, Data visualization and analysis of energy flow on a multi-zone building scale. Automation in Construction, 2017. 84: p. 258-273.

[66] Aste, N., M. Manfren, and G. Marenzi, Building Automation and Control Systems and performance optimization: A framework for analysis. Renewable and Sustainable Energy Reviews, 2017. 75: p. 313-330.

[67] https://www.buildingenergysoftwaretools.com/.

[68] Mahmud, K., et al., Computational tools for design, analysis, and management of residential energy systems. Applied Energy, 2018. 221: p. 535-556. 